\title{
Detection of sub-basaltic sediments by a multi-parametric joint inversion approach
}

\author{
Ajay Manglik*, Saurabh K Verma and K H Singh ${ }^{1}$ \\ National Geophysical Research Institute (Council of Scientific 8 Industrial Research), Hyderabad 500 606, India. \\ ${ }^{1}$ Present Address: GEST UMBC @ Planetary Geodynamics Laboratory, NASA Goddard Space Flight Center, \\ Greenbelt, Maryland, USA. \\ *e-mail: ajay@ngri.res.in
}

In many parts of the world sedimentary horizons with potential for hydrocarbon are located below flood basalt provinces. However, the presence of high velocity basaltic overburden makes delineation of sediments difficult due to the low velocity layer problem. Electrical and electromagnetic methods have been used in such scenarios because of the good electrical conductivity contrast between basalts and underlying sediments. However, mapping of the target sediments becomes difficult when the layer is thin as the data errors due to inherent noise lead to equivalent solutions. To tackle such difficult situations, a joint inversion scheme incorporating seismic reflection and refraction, magnetotelluric and deep electrical resistivity datasets is presented. Efficacy of the scheme is tested for a model comprising a thin sedimentary layer sandwiched between a thick basalt cover and a granitic basement. The results indicate that the parameters of the target sedimentary layer are either poorly resolved or equivalent solutions are obtained by the inversion of individual datasets. Joint inversions of seismic reflection (RFLS) and refraction (RFRS), or DC and MT dataset pairs provide improved results and the range of equivalent solutions is narrowed down. Combination of any three of the above datasets leads to further narrowing of this range and improvements in mean model estimates. Joint inversion incorporating all the datasets is found to yield good estimates of the structure. Resolution analysis is carried out to appraise estimates of various model parameters obtained by jointly inverting different combinations of datasets.

\section{Introduction}

In many parts of the world sedimentary horizons with potential for hydrocarbons are located below flood basalt provinces (Biswas 1982; Withers et al 1994; Milani et al 1990). However, the presence of the high velocity basaltic overburden and the underlying high velocity basement makes the seismic delineation of sandwiched low velocity sediments (LVL) difficult due to the absence of any refraction data from the top of the LVL and entrapment of energy in the form of reverberations within the LVL yielding feeble reflections at short offset distances. On the other hand, the electrical resistivity contrast between the basalts and sediments can be utilized to map the sediments using magnetotelluric (MT) and direct current (DC) resistivity methods (Morrison et al 1996). Despite the fact that these methods measure the response of the same physical property, they exploit basically different physical processes; thus resolving various geological units (traps, sediments, basement, etc.) differently (Verma and Sri Niwas 1994). Such differences become more prominent when fine (thin) sedimentary structures are to be delineated under moderate to thick basalts cover. In such situations, the problem of equivalence, i.e., different combinations of the values of resistivity and the thickness of a layer leading to apparent resistivity curves which, within the accuracy of

Keywords. Joint inversion; magnetotellurics; seismics; DC resistivity; exploration; geophysics; modelling; structure of the earth. 
the observation, are equal to each other (Koefoed 1969), also contributes significantly towards the ambiguity of interpretation (Verma and Sharma 1993; Withers et al 1994). For example, for DC resistivity methods, Inman (1975) has shown that equivalent solutions resolving the parametric combinations $h_{i} \rho_{i}$ (or $T_{i}$, implying resistivity $\left(\rho_{i}\right)$ and thickness $\left(h_{i}\right)$ product of the $i$ th layer) and $h_{i} / \rho_{i}$ (or $S_{i}$, implying conductivity and thickness product of the $i$ th layer) are obtained for thin resistive and conductive layers, respectively. For thin resistive sandwiched layers the equivalence gets prominent and $T_{i}$ is typically the best-resolved parameter. For MT method, the conductivity distribution can be determined uniquely in principle (Bailey 1970). However, data error and data insufficiency lead to non-uniqueness and $S_{i}$ is the bestresolved parameter while $T_{i}$ is resolved poorly. Joint inversion of DC and MT data becomes advantageous because they are sensitive to resistive and conductive structures respectively such that their combination enhances the resolution of $S_{i}$ and $T_{i}$.

In the case of seismic methods, the presence of overlying basalts makes the delineation of sediments difficult because the high seismic velocity of the basalts masks the seismic signatures of the underlying sediments. Though refraction seismics is widely employed in exploration geophysics, it fails to detect a sandwiched LVL. As no critically refracted waves are generated at the top of the LVL, there is an overestimation of the thickness of the overlying layer and consequently all the underlying layers. Seismic reflections from the top of the LVL could provide useful information but are often plagued by reverberations and high noise levels rendering it difficult to estimate the LVL parameters. Thus, it is observed that all the methods (seismic refraction and reflection; MT; and DC resistivity) have their own strengths and weaknesses in resolving different geological units.

Interpretation combining two or more datasets is undertaken in order to achieve improved estimates of the model parameters in such situations (Warren 1996; Satpal et al 2006). In this approach, the model parameters obtained by one method are used to constrain the model employed to interpret another dataset. The process can be repeated for a number of times such that finally a model more acceptable to both datasets is obtained. The joint inversion considers several datasets simultaneously by a numerical scheme that iteratively modifies a common starting model until an optimum model satisfying all datasets simultaneously within a specified error tolerance is obtained. Earlier, Manglik and Verma (1998) developed a one-dimensional joint inversion scheme combining MT and seismic reflection (RFLS) and refraction (RFRS) travel time data. Manglik and Verma
Table 1. Representative geological model used in the analysis.

\begin{tabular}{lccc}
\hline & \multicolumn{3}{c}{ True model } \\
\cline { 2 - 4 } Geological unit & $\rho(\Omega . \mathrm{m})$ & $h(\mathrm{~m})$ & $\nu(\mathrm{km} / \mathrm{s})$ \\
\hline Trap & 250 & 800 & 4.5 \\
Sediments & 15 & 250 & 2.7 \\
Basement & 5000 & $\propto$ & 6.0 \\
\hline
\end{tabular}

(1999) included DC resistivity inversion to their joint MT - seismic inversion algorithm. Hering et al (1995) reported an example of combining DC electrical data with surface wave data. Recently, Gallardo and Meju (2007) presented an algorithm for the joint inversion of MT and seismic refraction data for two dimensional structures.

This paper presents a detailed mathematical formulation of the joint inversion algorithm by Manglik and Verma $(1998,1999)$ to model RFLS, RFRS, MT, and DC data simultaneously for a layered earth model. The algorithm is designed in such a way that any combination of datasets (viz., DC-RFLS, MT-RFLS, MT-DC-RFLS, MT-DCRFRS-RFLS, etc.) can be considered for joint inversion. Numerical experiments are conducted for a representative geological model (table 1) of sub-basalt sediments to examine their resolution by various combinations of datasets. Since a thick layer of sediments makes an easy target to resolve, a relatively more difficult target of $250 \mathrm{~m}$ thick sediments, sandwiched between an overburden layer of $800 \mathrm{~m}$ thick basalts and a granitic basement, is considered here.

\section{Formulation}

Although basic formulations for a one-dimensional multi-layered earth are presented in a number of papers and are also available in some standard books, a few pertinent expressions used in the present analysis are given below.

\subsection{DC resistivity}

We consider only Schlumberger sounding here for which the apparent resistivity, $\rho_{a s}$, at a distance $r$ (or $A B / 2$, half the current electrode spacing) is given as (Ghosh 1971; Inman 1975; Vozoff and Jupp 1975):

$$
\rho_{a s}(r)=r^{2} \int_{0}^{\infty} T(\lambda) \lambda J_{1}(\lambda r) d \lambda,
$$

where $J_{1}$ is the Bessel function of first order and the resistivity transform, $T(\lambda)$ is given by the 
recurrence relation:

$$
\begin{aligned}
T_{i-1}(\lambda)= & \frac{T_{i}(\lambda)+\rho_{i-1} \tanh \left(\lambda h_{i-1}\right)}{1+T_{i}(\lambda) \tanh \left(\lambda h_{i-1}\right) / \rho_{i-1}}, \\
& (i=1, \ldots, n-1) .
\end{aligned}
$$

In the above equation $\rho_{i}$ and $h_{i}$ are the resistivity and thickness of the $i$ th layer and $T_{n}(\lambda)=$ $\rho_{n}$ which implies that for an $n$-layered earth, the resistivity transform of the $n$th layer equals the true resistivity of the half-space.

\subsection{Magnetotellurics}

For a multi-layered earth, the surface impedance $Z_{1}$ as a function of frequency $\omega$ can be written as (Vozoff and Jupp 1975):

$$
\rho_{a}(\omega)=\frac{1}{|w|} Z_{1}^{*} Z_{1}
$$

where $w=(-i \omega \mu)^{1 / 2}$ and $Z_{1}^{*}$ is the complex conjugate of the impedance $Z_{1}, \omega$ is the angular frequency and $\mu$ is magnetic permeability of the medium. Once again $Z_{1}$ can be computed using the following recurrence relation:

$$
\begin{gathered}
Z_{i}(\omega)=\frac{\left(Z_{i+1}+T_{i}\right)}{\left(1+S_{i} Z_{i+1}\right)}, \quad(i=1, \ldots, n-1), \\
\quad \text { and } \quad Z_{n}=w \sqrt{\rho_{n}} .
\end{gathered}
$$

where

$$
\begin{aligned}
T_{i} & =\left(w \sqrt{\rho_{i}}\right) \tanh \left(\frac{w h_{i}}{\sqrt{\rho_{i}}}\right), \\
S_{i} & =\frac{1}{w \sqrt{\rho_{i}}} \tanh \left(\frac{w h_{i}}{\sqrt{\rho_{i}}}\right) .
\end{aligned}
$$

The partial derivatives of $\rho_{a s}(r)$ and $\rho_{a}(\omega)$ for DC and MT cases are computed by differentiating equations (1) and (3) with respect to layer parameters. Since these are well known (e.g., Inman 1973; Jupp and Vozoff 1975; Vozoff and Jupp 1975) the expressions are not given here.

\subsection{Seismic refraction and reflection}

To compute the refraction travel time, we take the following expression (Sain and Kaila 1996; Manglik and Verma 1998) for a horizontally layered, homogeneous, isotropic earth:

$$
\begin{gathered}
T_{j+1}^{r f r}=\frac{X}{\nu_{j+1}}+2 \sum_{k=1}^{j} \frac{h_{k}}{\nu_{j+1} \nu_{k}}\left[\nu_{j+1}^{2}-\nu_{k}^{2}\right]^{1 / 2}, \\
(j=1, \ldots, n-1),
\end{gathered}
$$

where $T_{j+1}^{r f r}$ is the time taken by the head wave from $(j+1)$ th interface to reach the surface at an offset distance of $X, v_{j+1}$ is the seismic velocity of the $(j+1)$ th layer, and $h_{k}$ and $v_{k}$ are the thickness and velocity of the $k$ th layer.

The travel time for the reflection from the $j$ th interface, $T_{j}^{r f l}$, at offset distance $x$ can be calculated by using (Sain and Kaila 1994; Manglik and Verma 1998):

$$
\begin{gathered}
T_{j}^{r f l}=2 \sum_{k=1}^{j} \frac{h_{k}}{\nu_{k}\left(1-R^{2} \nu_{k}^{2}\right)^{1 / 2}}, \\
(j=1, \ldots, n-1),
\end{gathered}
$$

where the ray parameter, $R$, can be obtained using the following equation at every geophone location $x$ :

$$
x=2 R \sum_{k=1}^{j} \frac{h_{k} \nu_{k}}{\left(1-R^{2} \nu_{k}^{2}\right)^{1 / 2}} .
$$

In equations (7) and (8), $R$ is crucial as it controls the ray path and is required in the computations of the partial derivatives. Since the expressions of partial derivatives for seismic reflection and refraction cases are discussed in Manglik and Verma (1998), these are not given here.

\section{Inversion approach}

An iterative linearised inversion scheme, based on the Jacobian or the partial derivative matrix, is used (Marquardt 1970). Let us assume that $G_{D C}$, $G_{M T}, G_{R F L}$ and $G_{R F R}$ are the observed responses for DC, MT, seismic reflection and refraction methods, respectively. Further, let $D_{D C}, D_{M T}$, $D_{R F L}$, and $D_{R F R}$ be the number of data points at which $\rho_{a s}(r)$ at various current electrode spacings, $\rho_{a}(\omega)$ for various frequencies, reflection and 
refraction travel times at different offset distances are observed. For the unknown model parameters $P_{j} \quad(j=1,2, \ldots, 3 n-1 ; n$ being the number of layers including half space) the equations governing the inversion process for the four methods can be written as:

$$
\begin{aligned}
& \left\{\Delta G_{D C}\right\}=\left[\begin{array}{ll}
A_{D C}^{\rho} & A_{D C}^{h}
\end{array}\right] \cdot\left\{\begin{array}{c}
\Delta \rho \\
\Delta h
\end{array}\right\}, \\
& \left(D_{D C} \times 1\right)\left(D_{D C} \times(2 n-1)\right)((2 n-1) \times 1), \\
& \left\{\Delta G_{M T}\right\}=\left[\begin{array}{ll}
A_{M T}^{\rho} & A_{M T}^{h}
\end{array}\right] \cdot\left\{\begin{array}{c}
\Delta \rho \\
\Delta h
\end{array}\right\}, \\
& \left(D_{M T} \times 1\right)\left(D_{M T} \times(2 n-1)\right)((2 n-1) \times 1), \\
& \left\{\begin{array}{ll}
\Delta G_{R F L}
\end{array}=\left[\begin{array}{ll}
A_{R F L}^{h} & A_{R F L}^{v}
\end{array}\right] \cdot\left\{\begin{array}{c}
\Delta h \\
\Delta v
\end{array}\right\},\right. \\
& \left(D_{R F L} \times 1\right)\left(D_{R F L} \times(2 n-2)\right)((2 n-2) \times 1),
\end{aligned}
$$

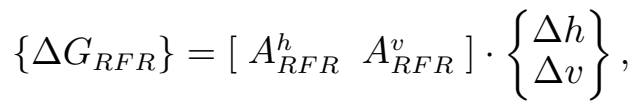

$$
\begin{aligned}
& \left(D_{R F R} \times 1\right)\left(D_{R F R} \times(2 n-1)\right)((2 n-1) \times 1),
\end{aligned}
$$

where the $A$ 's form partial derivative (Jacobian) matrices with respect to model parameters, and $\Delta \rho, \Delta h, \Delta v$ constitute the model correction vector $\Delta P$.

Since the observations by different methods and various model parameters have different physical dimensions, the elements of the above partial derivative matrices (equation 9) have different dimensional units. Therefore, these matrices have been converted into non-dimensional form. For this purpose, a reference model was chosen and various forward responses were computed. Normalization of observed responses and model parameters was done with respect to this reference model. The reference model can be arbitrarily chosen but we have considered the model of previous iteration as our reference model for the next iteration (Manglik and Verma 1998).

Equation (9) can be combined into a single matrix equation

$$
\begin{gathered}
\left\{\begin{array}{c}
\Delta G_{D C} \\
\Delta G_{M T} \\
\Delta G_{R F L} \\
\Delta G_{R F R}
\end{array}\right\}= \\
(D \times 1) \quad\left[\begin{array}{ccc}
A_{D C}^{\rho} & A_{D C}^{h} & 0 \\
A_{M T}^{\rho} & A_{M T}^{h} & 0 \\
0 & A_{R F L}^{h} & A_{R F L}^{v} \\
0 & A_{R F R}^{h} & A_{R F R}^{v}
\end{array}\right] \cdot\left\{\begin{array}{c}
\Delta \rho \\
\Delta h \\
\Delta v
\end{array}\right\} . \\
(D \times(3 n-1)) \quad((3 n-1) \times 1)
\end{gathered}
$$

In a concise form the above equation is expressed as:

$$
\Delta G=A_{c} \cdot \Delta P
$$

where

$$
\Delta P=\left\{\begin{array}{lll}
\Delta \rho & \Delta h & \Delta v
\end{array}\right\}^{T} .
$$

In the above equation, $\Delta G$ denotes the $(D \times 1)$ column vector $\left(D=D_{D C}+D_{M T}+D_{R F L}+D_{R F R}\right)$, $A_{c}$ is the combined partial derivative matrix of dimension $D \times(3 n-1)$ and $\Delta P$ is the model correction vector of dimension $(3 n-1) \times 1$. We solve the above equation by using a SVD solver to minimize the root mean square error, $\sigma$, given by:

$$
\sigma=\sqrt{\frac{1}{D} \sum_{i=1}^{D} \Delta G_{i}^{2}}
$$

An additional criterion of model misfit function, $M M F$, satisfying the following condition:

$$
M M F=\sqrt{\frac{1}{3 n-1} \sum_{j=1}^{3 n-1} \Delta P_{j}^{2}},
$$

has also been used in the inversion analysis.

\subsection{Singular value decomposition analysis}

The Jacobian matrix $A_{c}$ can be decomposed into $U, V$ and $\Lambda$ matrices using SVD. Thus

$$
A_{c}=U \Lambda V^{T},
$$

where $U$ and $V$ are orthogonal set of data and model space eigen-vectors having dimension $D \times(3 n-1)$ and $(3 n-1) \times(3 n-1)$, respectively. $\Lambda$ is a matrix with diagonal elements having singular-values, $\lambda_{i}(i=1, \ldots, 3 n-1)$. For an overdetermined problem, $A_{c}$ is rank deficient, and the spaces $U$ and $V$ can then be divided into subspaces $\left(U_{P}, V_{P}\right)$ spanned by data and model eigenvectors associated with $p(p \leq \min (D, 3 n-1))$ nonvanishing eigen-values $\Lambda_{P}$ and the subspaces $\left(U_{0}, V_{0}\right)$ spanned by eigen-vectors associated with zero eigen-values. The generalized inverse of the matrix $A_{c}$ can be expressed as:

$$
A^{+}=V_{P} \Lambda_{P}^{-1} U_{P}^{T},
$$


where the matrix $\Lambda_{P}^{-1}$ is the inverse of the diagonal matrix $\Lambda_{P}$. The solution of the matrix equation (11) is obtained by:

$$
\Delta \hat{P}=A^{+} \Delta G
$$

where $\Delta \hat{P}$ is the estimated model parameter vector.

The field data is generally corrupted with noise. Hence, it becomes necessary to study how the noise in data gets mapped into errors in model estimates. If we assume that each datum has a Gaussian noise with $\sigma_{d}$ standard deviation, the error in the solution $\Delta \hat{P}$ can be written as (Menke 1989):

$$
[\operatorname{cov} \Delta \hat{P}]=A^{+}[\operatorname{cov} \Delta G]\left(A^{+}\right)^{T},
$$

where $[\operatorname{cov} \Delta \hat{P}]$ and $[\operatorname{cov} \Delta G]$ are the covariance matrices of estimated parameter change vector $\Delta \hat{P}$ and the actual data difference vector $\Delta G$. For an over-determined case, when number of data point exceeds number of model parameter, the covariance can be written as

$$
[\operatorname{cov} \Delta \hat{P}]=\sigma_{d}^{2}\left[A_{c}^{T} A_{c}\right]^{-1}
$$

In equation (14) it is seen that the matrix $A_{c}$ can be decomposed in the form of eigen-vectors corresponding to non-zero eigen-values and those representing zero eigen-values and an inverse of $A_{c}$ can be obtained as given by equation (15). However, for cases when some of the eigen-values become very small, as mostly encountered in geophysical problems, the inverse of $A_{c}$ becomes very sensitive to noise. Although, there are some approaches suggested for the solution of $A_{c}$ matrix by enhancing its diagonal elements (Marquardt 1970), in SVD approach a cut-off value $\varepsilon$, given as:

$$
\varepsilon \geq \frac{\lambda_{i}}{\lambda_{\max }}
$$

is used to ignore all the eigen-values smaller than this cut-off value. This constraint stabilizes the solution and controls the error propagation but sacrifices the resolution. The value of $\varepsilon$ can be reduced at a later stage of iteration when the solution starts converging to a final model. Backus and Gilbert (1970) graphically demonstrated that in the case of noisy data the error could not be minimized below a certain cut-off value as it results in an increase in the standard deviation of obtained model parameters.

\section{Results}

To study the performance of the multi-parametric joint inversion scheme, synthetic responses for a representative geological model (table 1) were generated for DC, MT, RFLS and RFRS methods.

To simulate the field situation, we corrupted these data with various levels of noise to mimic field data. DC data were corrupted with $10 \%$ uniform random noise, and MT with a linearly increasing noise of 10 to $15 \%$ over the period range $10^{-2}$ $10^{2} \mathrm{~s}$. The high noise at longer periods was introduced to simulate realistic field conditions. We have considered only the amplitude data for MT method because the phase data with this amount of noise were found to deteriorate the solution. The RFLS data were contaminated with a noise of 70-50 millisecond (ms) linearly decreasing with the offset distance. Again this was done to represent high noise level in reflection data at shorter offset distances. The RFRS data were corrupted with a uniform random noise of $60 \mathrm{~ms}$. The DC, MT, RFLS, and RFRS data thus obtained, are shown in figure 1 by symbols. To characterize the data completely, a scatter or standard deviation with Gaussian distribution was also added to every noisy (represented by symbols) data point. Standard deviation of $10 \%$ was added to DC and MT data, while seismic data exhibit a scatter of $60 \mathrm{~ms}$. Synthetic noise-free responses are shown by continuous line (dashed line in case of RFRS).

After the preparation of various datasets as described above, inversions were performed for all the methods considering different initial guesses. Computations and analyses of results were carried out in four steps. First, only the individual inversions of DC, MT and RFLS data were undertaken to study the performance of individual methods. Results for the inversion of RFRS data alone are not presented, as it does not provide any information on the LVL. Next, joint inversions were carried out for similar pairs of datasets, i.e., MT and DC, and RFLS and RFRS (designated as SI). This was followed by the joint inversion considering three datasets at a time (i.e., DC-SI and MT-SI). Finally all four datasets (DC, MT, and SI) were considered together to study the improvement in the estimation of model parameters. The results are given in table 2 .

\subsection{Inversion of individual datasets}

Results for this case for three initial guesses IG-1, IG-2, and IG-3, are shown in rows against DC, MT, and RFLS in table 2. The DC data are able to estimate the parameters of the top layer (basaltic cover) reasonably well regardless of the choice of the initial model. However, the 

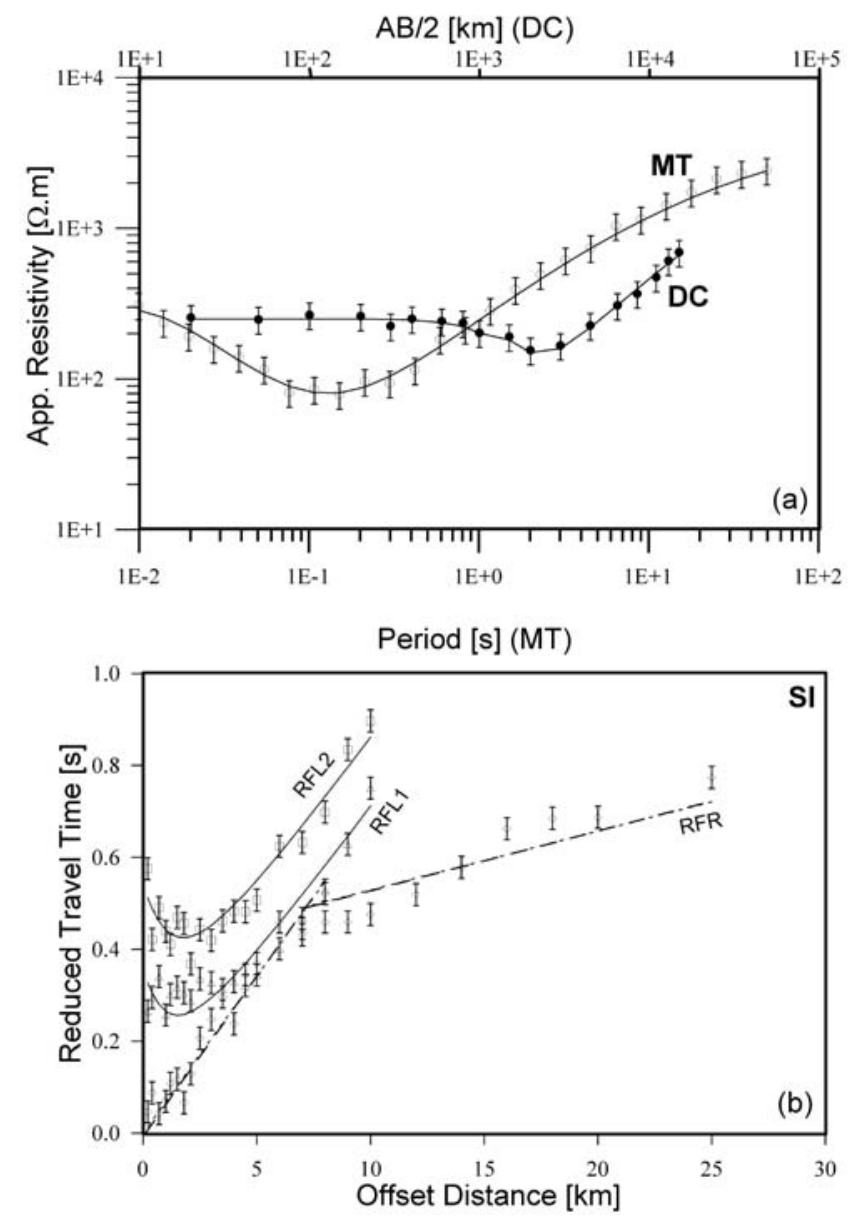

Figure 1. (a) Direct current resistivity and magnetotelluric data, and (b) reflection and refraction seismic data used in the analysis. Smooth curves represent synthetic response and symbols show the noisy data.

parameters obtained for the sedimentary layer are strongly dependent on the choice of the initial guess and in general equivalent solutions $\left(S_{2}\right.$ constant) are obtained. These initial guess models also give acceptable values for the resistivity of the basement. Results corresponding to the MT data again show a strong dependence on the choice of initial guess model. The initial guess model IG-2 gives somewhat better results compared to those obtained from IG-1 and IG-3. Estimates for the basaltic layer are generally poor (except for IG-2) and equivalent solutions ( $S_{2}$ constant) for the sedimentary layer are obtained. All the three initial guess models provide acceptable values for the resistivity of the basement. For the reflection seismic (RFLS) data alone only the top two layers can be studied, as the reflections from the bottom of the basement are not available. Slightly improved estimates of various parameters are obtained for IG-2. RFLS inversion provides good estimates for the basaltic overburden while equivalent solutions $\left(h_{2} / v_{2}\right.$ constant $)$ are obtained for the sedimentary layer.

\subsection{Joint inversion of similar dataset pairs}

Next, similar datasets (MT-DC and RFLS-RFRS) were inverted jointly to study improvements in the mean estimates of model parameters. It is found that MT-DC results are by and large independent of the initial model. An overall improvement in estimates of various parameters is observed. Parameters of the sedimentary layer still show equivalence but their mean estimates are closer to the true values. Joint inversion of RFLS-RFRS data (designated as SI in table 2) provides only minor improvement over the results obtained by the individual inversion of RFLS data. Addition of RFRS data nevertheless provides information on the velocity of the basement.

\subsection{Joint inversion of three datasets}

Combination of DC and seismic (SI) information, and MT and SI information is studied next. These are shown as MT-SI and DC-SI in table 2 for different initial models. Results get more stable (less dependent on the choice of initial model) and show further improvement in the mean estimates of various model parameters. Though the problem of electrical and seismic equivalence (constant $S_{2}$ and $h_{2} / v_{2}$, respectively) for the sediments still persists, it is considerably reduced and the mean estimates of $\rho_{2}, h_{2}$ and $v_{2}$ are improved. It can also be seen that MT-SI inversion yields slightly better results in comparison to DC-SI results.

\subsection{Joint inversion of all datasets}

Finally the joint inversion of all the four datasets, i.e., DC-MT-SI (designated as JI in table 2), is done to study the overall improvement in mean parameter estimates. Very good estimates of all parameters are obtained in this case for all initial guess models. Mean estimates of various parameters are very close to their true values. These results suggest that the problem of equivalence associated with the sedimentary layer is reduced to a great extent. Thus the joint inversion of all datasets is desirable.

\section{Sensitivity and resolution analysis}

In the preceding section, the results were analyzed on the basis of closeness of the estimated mean values to the true values of various model parameters. However, it is important to study the sensitivity of model parameters to noise to assess the reliability of their estimated values. For this purpose, we have analyzed eigen-values and model eigen-vectors using the results from SVD analysis for all the three initial guess models. Since these 


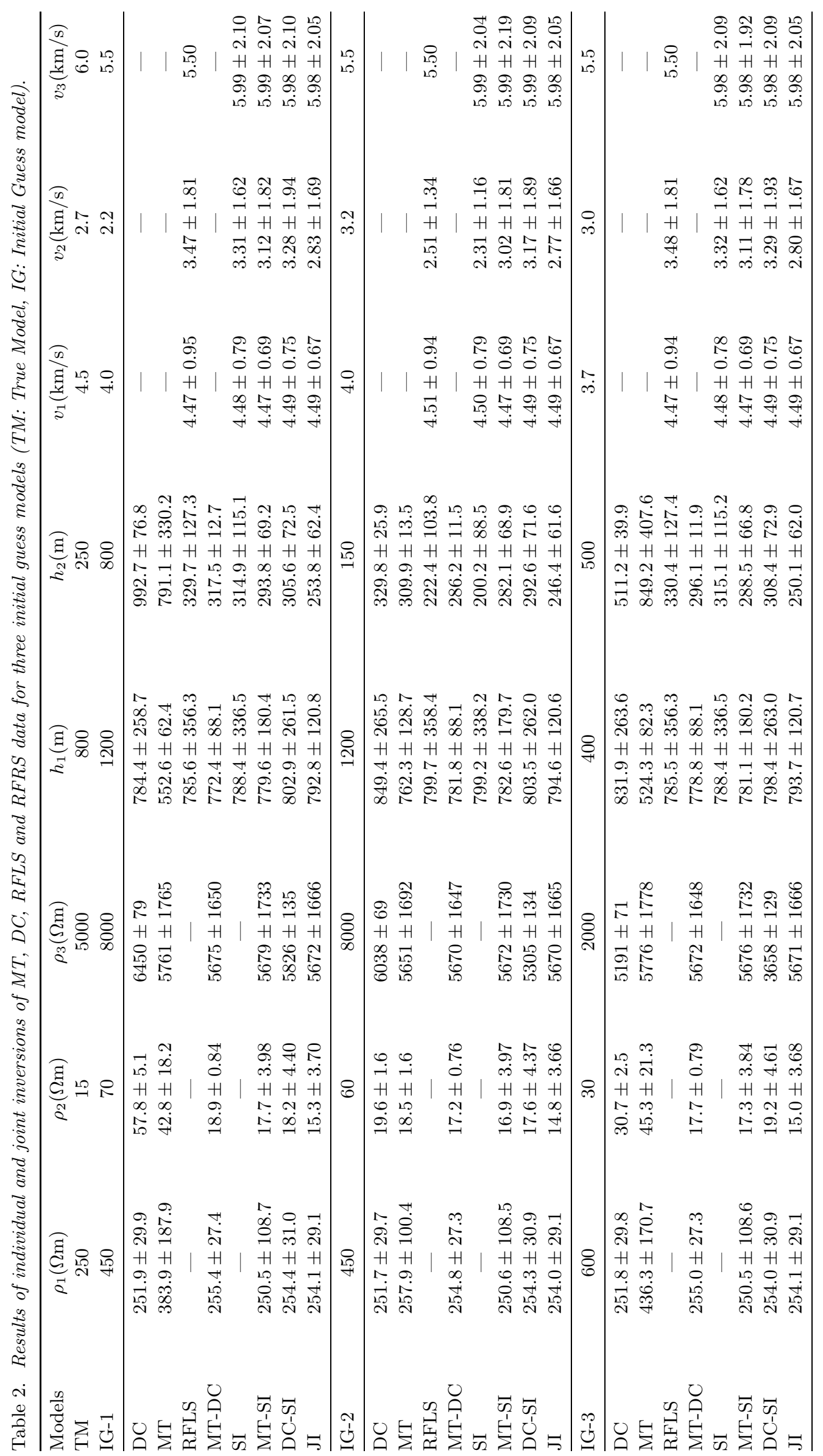




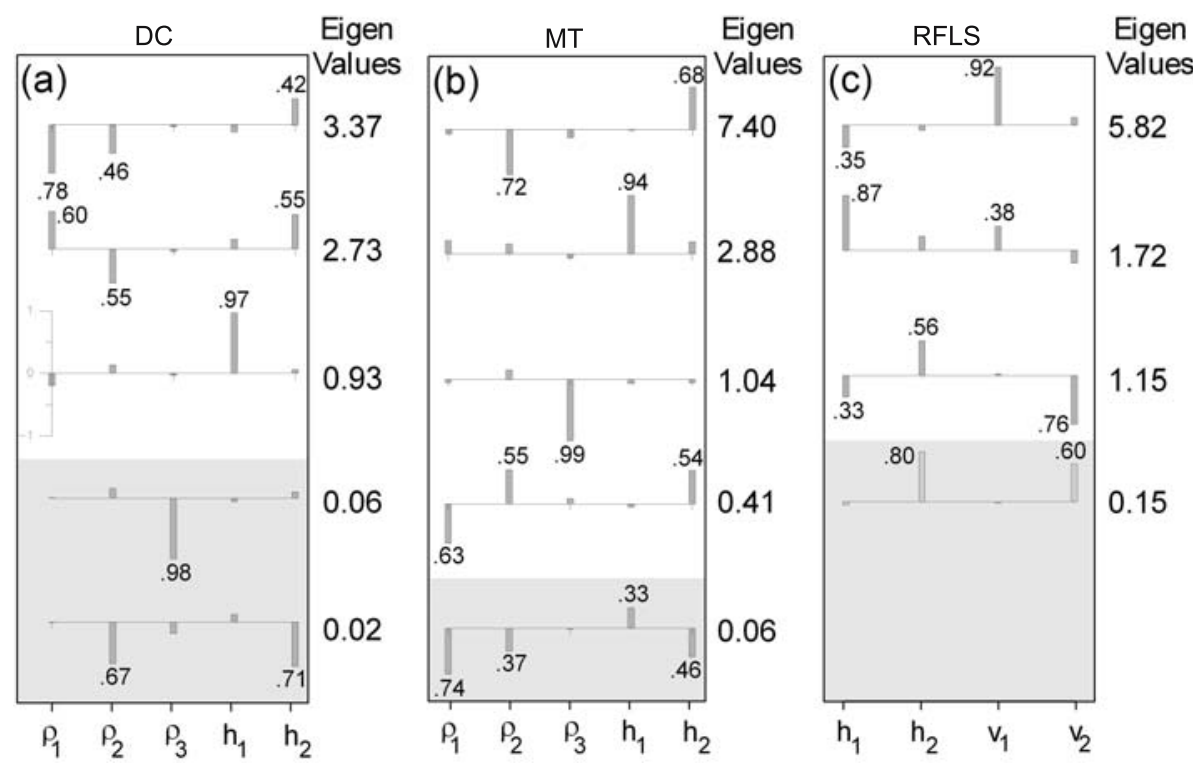

Figure 2. Model eigen-vector analysis for (a) DC, (b) MT, and (c) RFLS dataset for the initial guess model IG-1.

results are almost similar (with very little differences), only those corresponding to IG-1 are presented and discussed here. We follow an approach of using parameter eigen-vectors, also called V-matrix analysis, to analyze the resolution and sensitivity of estimated model parameters (Inman 1973; Sharma 1994) rather than discussing the resolution in terms of parameter resolution matrix $R=V V^{T}$ (Menke 1989). The analysis of eigen-vectors yields additional information on the stability of the solution which can be seen in the example given in section 5.1 for which parameter resolution matrix is also presented.

\subsection{Individual datasets}

For the individual datasets (DC, MT and RFLS), the results are shown in figure 2 . It can be seen that for the DC data (figure 2a) the highest eigen-value is associated with the parametric combination $h_{2}^{0.42} /\left(\rho_{1}^{0.78} \cdot \rho_{2}^{0.46}\right)$ suggesting that this combination is least sensitive to noise. Further, the parameter $\rho_{1}$ is estimated reasonably well while $S_{2}$ is estimated moderately well. The next best estimated combination is $\rho_{1}^{0.6} \cdot h_{2}^{0.55} / \rho_{2}^{0.55}$. The thickness of the basalts, $h_{1}$, is associated with the third significant eigen-value. These are the only parameters that are resolved well by the DC data. Other parametric combinations are associated with very small eigen-values that were ignored in the inversion. These are shown by the shaded region in figure 2(a). Thus, for example, the basement resistivity, $\rho_{3}$, is associated with a very small eigenvalue and therefore it is very sensitive to noise. Even though good mean estimates of the parameter $\rho_{3}$ are obtained from DC data (table 2), it is a poorly resolved parameter according to V-matrix analysis. Results for MT data (figure 2b) provide resolution of the following parametric combinations in descending order: $h_{2}^{0.68} / \rho_{2}^{0.72}$ (or $\left.S_{2}\right), h_{1}, \rho_{3}$, and $\rho_{2}^{0.55} \cdot h_{2}^{0.54} / \rho_{1}^{0.63}$. Thus it can be seen that the MT data best resolves the parameter $S_{2}$. Estimates for $\rho_{3}$ are also more reliable in comparison to that obtained by DC data. The RFLS data (figure 2c) resolve parameters in the following order: $v_{1}^{0.92} / h_{1}^{0.35}, h_{1}^{0.87} \cdot v_{1}^{0.38}$, and $h_{2}^{0.56} /\left(h_{1}^{0.33} \cdot v_{2}^{0.76}\right)$. Thus the parameters $v_{1}$ and $h_{1}$ are resolved well while the parameters of the sedimentary layer are resolved as the ratio $h_{2} / v_{2}$. It may be noted that for the sediments the RFLS data gives higher weight to the velocity parameter $\left(v_{2}\right)$ in comparison to the thickness parameter $\left(h_{2}\right)$.

Figure 3, shows parameter resolution matrix for the above models. For the DC case (figure 3a), only parameters $\rho_{1}$ and $h_{1}$ are well resolved and the resistivity $\rho_{3}$ is not resolved. The resolution of parameters for MT case (figure $3 \mathrm{~b}$ ) is much better. In RFLS (figure 3c), only the first layer

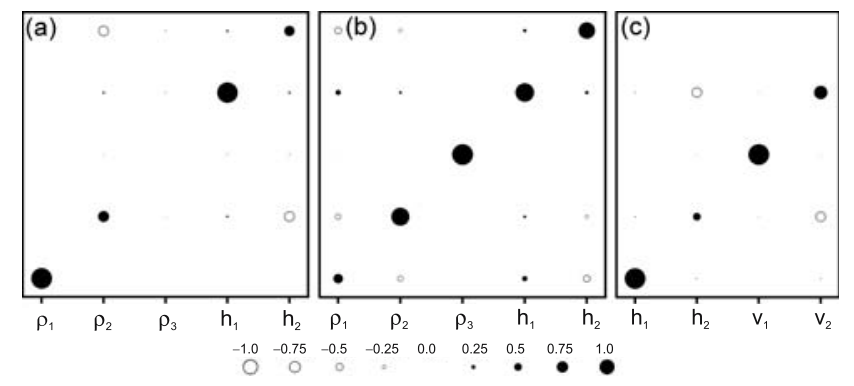

Figure 3. Parameter resolution matrix for (a) DC, (b) MT, and (c) RFLS dataset for the initial guess model IG-1. 

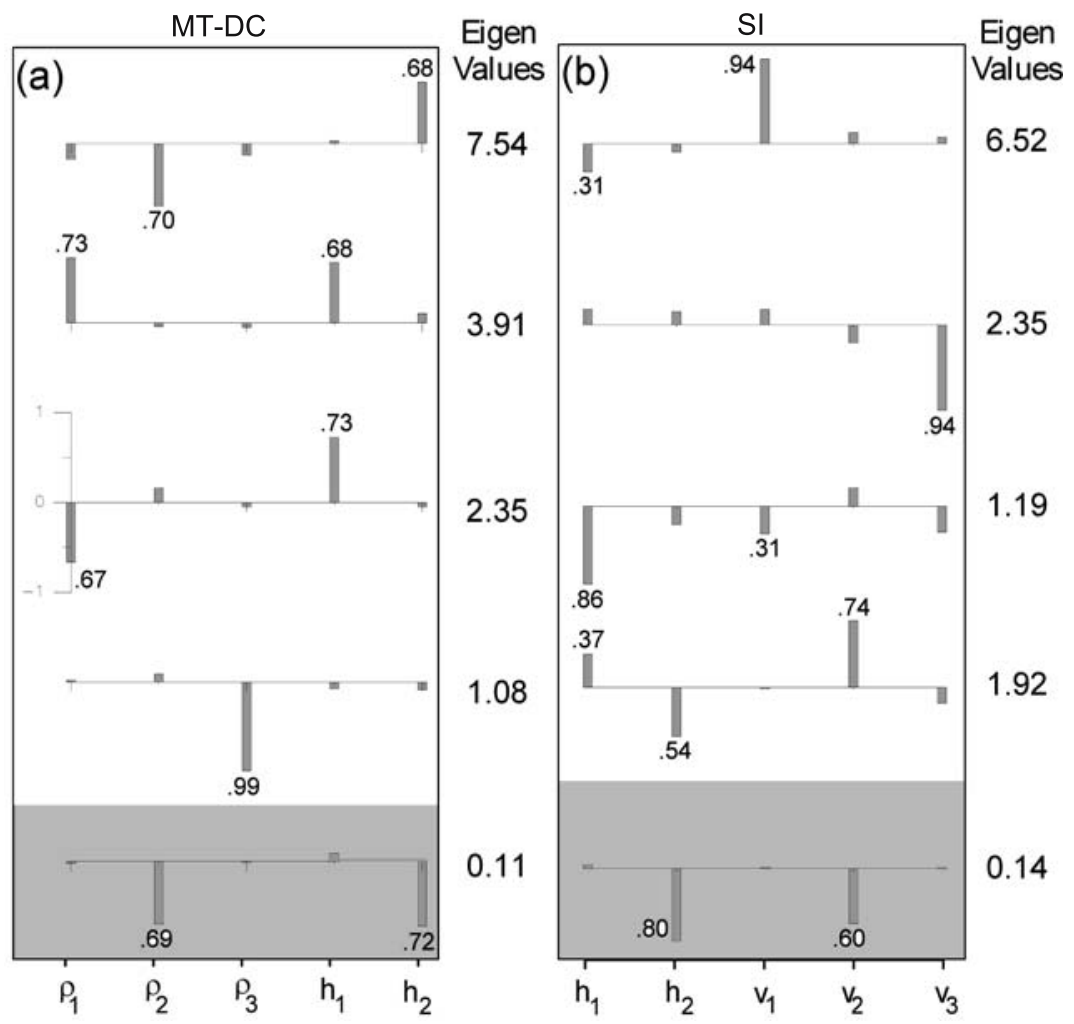

Figure 4. Model eigen-vector analysis for (a) MT-DC and (b) SI dataset for the initial guess model IG-1.

parameters are well resolved. The parameter resolution matrix, however, gives information about the resolvability of the parameters for the inverted model without providing any further information about the stability of the estimated parameters. For example, by including one more eigen-value $\left(\lambda_{5}=0.06\right.$ in figure $\left.2 \mathrm{a}\right)$ in the DC model, we would have obtained a corresponding parameter resolution matrix showing $\rho_{3}$ as a well resolved parameter without any information about its sensitivity to noise. On the other hand, V-matrix analysis shows that this parameter $\rho_{3}$ is sensitive to the noise in data. Therefore, V-matrix analysis can help in selecting a trade-off between the resolution and stability of the estimated model parameters. Therefore, we discuss only V-matrix analysis in subsequent sections.

\subsection{Similar datasets}

Next, the resolutions obtained by the combination of two similar datasets (MT-DC and RFLSRFRS) are studied (figure 4). The four significant eigen-values for the MT-DC joint inversion (figure 4a) resolve the following parameters: $h_{2}^{0.68} / \rho_{2}^{0.7}, \rho_{1}^{0.73} \cdot h_{1}^{0.68}, h_{1}^{0.73} / \rho_{1}^{0.67}$, and $\rho_{3}$. Thus, the joint inversion of MT and DC datasets best resolves the parameter $S_{2}$. The second best resolved parameter is $T_{1}$. It may be noted here that while in principle the problem of equivalence (constant $S_{i}$ and $T_{i}$ ) can be reduced considerably by the joint inversion of $\mathrm{MT}$ and $\mathrm{DC}$ datasets, the V-matrix analysis shows that the resistivity and thickness parameters are still coupled. This can be ascribed to the magnitude of noise we have considered in the analysis. Nevertheless, from table 2 it is clear that the mean estimates of various model parameters show an overall improvement and the problem of equivalence (constant $S_{2}$ ) associated with the sedimentary layer is reduced significantly. The joint inversion of RFLS-RFRS data show (figure $4 \mathrm{~b}$ ) that the parameters are resolved in the following order: $h_{1}^{0.31} / v_{1}^{0.94}, v_{3}, 1 /\left(h_{1}^{0.86} \cdot v_{1}^{0.31}\right)$, and $h_{1}^{0.37} \cdot v_{2}^{0.74} / h_{2}^{0.54}$. Thus while the parameters of the basaltic overburden, $h_{1}$ and $v_{1}$, are resolved well, the parameters of the sediments are still resolved in the ratio $h_{2} / v_{2}$ with $v_{2}$ at a higher weight. The mean estimates of $h_{2}$ and $v_{2}$ (table 2) suggest a slight reduction in the problem of equivalence associated with the sediments. The velocity of the basement, $v_{3}$, is also resolved by the combination of RFLS and RFRS datasets.

\subsection{Three datasets}

Results for three datasets (DC-SI and MT-SI) are shown in figure 5. For DC-SI case (figure 5a), the largest eigen-value is associated with the parameter $v_{1}^{0.93} / h_{1}^{0.33}$ indicating that the velocity of basalts is the best-resolved parameter. Other parametric 


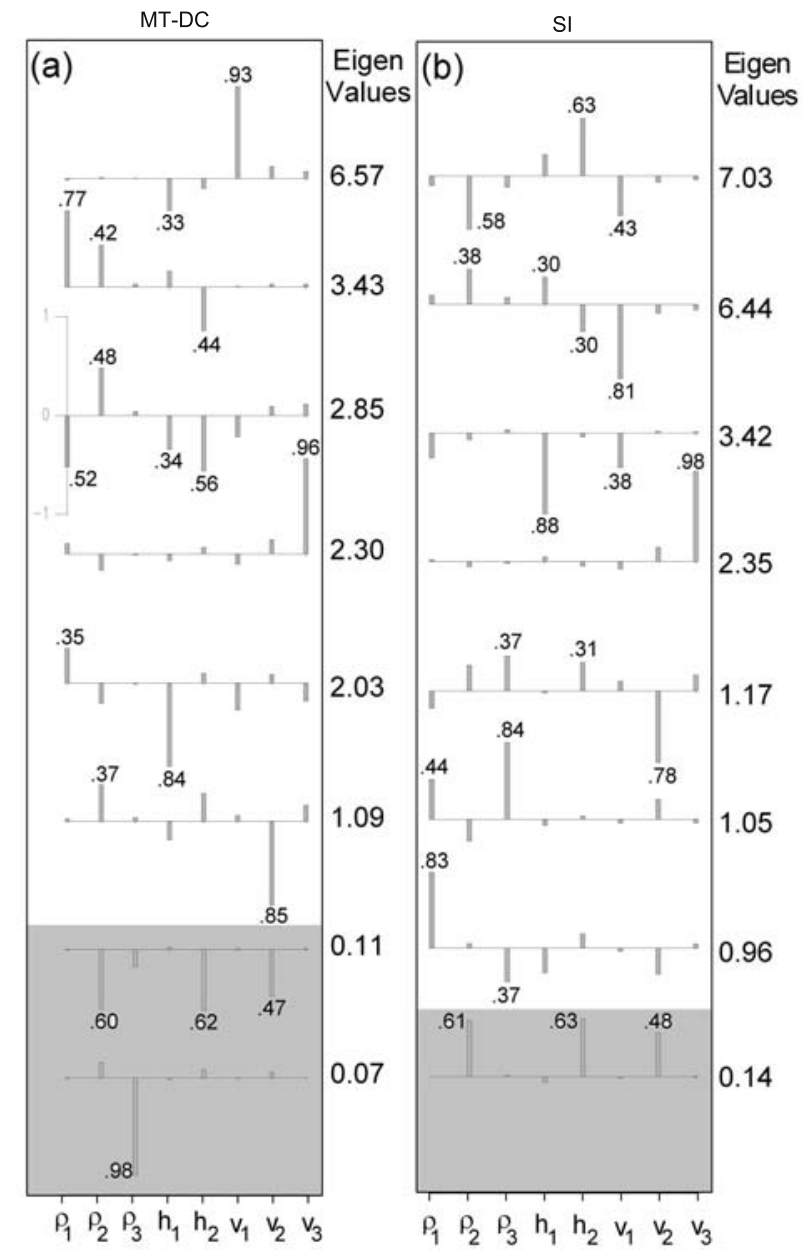

Figure 5. Model eigen-vector analysis for (a) DC-SI and (b) MT-SI dataset for the initial guess model IG-1.

combinations are resolved in descending order as follows: $\rho_{1}^{0.77} \cdot \rho_{2}^{0.42} / h_{2}^{0.44}, \rho_{2}^{0.48} /\left(\rho_{1}^{0.52} h_{1}^{0.34} h_{2}^{0.56}\right), v_{3}$, $\rho_{1}^{0.35} / h_{1}^{0.84}$ and $\rho_{2}^{0.37} / v_{2}^{0.85}$. Thus the DC-SI combination also resolves the parameters $\rho_{1}, v_{3}$, and $h_{1}$. However, parameters of the sedimentary layer are obtained only in terms of ratios indicating the problem of equivalence associated with the layer. The smallest significant eigen-value is associated with $v_{2}$ at a high weight. For the MT-SI combination (figure $5 \mathrm{~b}$ ), the order of resolution for various parametric combinations is: $h_{2}^{0.63} / \rho_{2}^{0.58} v_{1}^{0.43}$, $\rho_{2}^{0.38} h_{1}^{0.3} / h_{2}^{0.3} v_{1}^{0.81}, 1 /\left(h_{1}^{0.88} \cdot v_{1}^{0.38}\right), v_{3}, \rho_{2}^{0.37} \cdot h_{2}^{0.31} j$ $v_{2}^{0.78}, \rho_{1}^{0.44} \cdot \rho_{3}^{0.84}$, and $\rho_{1}^{0.83} / \rho_{2}^{0.37}$. Thus the parameters $v_{1}, h_{1}, v_{3}, \rho_{3}$ and $\rho_{1}$ are resolved well while the parameters of the sediments yield equivalent results. The parameter $v_{2}$ with high weight is associated with the fifth largest eigen-value.

\subsection{Four datasets}

Results for the combination of all the parameters (DC-MT-SI) are shown in figure 6. In this case various parametric combinations are resolved in the following order: $h_{2}^{0.68} / \rho_{2}^{0.66}, h_{1}^{0.38} / v_{1}^{0.88}$,
$1 /\left(\rho_{1}^{0.62} h_{1}^{0.69} v_{1}^{0.36}\right), \rho_{1}^{0.76} / h_{1}^{0.59}, v_{3}, \rho_{3}^{0.67} \cdot h_{2}^{0.31} / v_{2}^{0.63}$, and $\rho_{2}^{0.3} /\left(\rho_{3}^{0.73} \cdot h_{2}^{0.57}\right)$. Here the highest eigen-value is associated with the ratio $h_{2} / \rho_{2}$. Other resolved parameters are: $v_{1}, \rho_{1}, v_{3}$, and $\rho_{3}$. While the $\mathrm{V}$-matrix analysis suggests that the parameters of the sedimentary layer are coupled in the form of ratios to yield equivalent solutions, the mean estimated values of various parameters (table 2) lie very close to the true values of the parameters.

\section{Conclusions}

A general numerical scheme is developed to facilitate joint inversion of DC, MT, RFLS and RFRS datasets. It has the option to consider individual inversion of a single dataset or perform joint inversion considering any combination of two, three or four datasets. Efficacy of the scheme is established by considering a difficult situation encountered in the delineation of a thin sedimentary layer below a thick high velocity overburden of basalts. Performance of the scheme is studied for synthetic noisy datasets and results are analyzed in terms of the mean parameter estimates and resolution obtained by $\mathrm{V}$-matrix analysis for various cases.

While the inversion of individual datasets yields estimates that change with the choice of initial model, the joint inversion yields stable solutions that do not vary significantly with the initial model. Joint inversion of similar datasets (MT-DC and RFLS-RFRS) provides a good resolution of the resistivity and velocity of the basement. Parameters of the sub-basaltic sediments are most difficult to resolve and in general equivalent solutions in terms of constant $S_{2}$ and $v_{2} / h_{2}$ are obtained. However, it is observed that as more datasets are included in the joint inversion, the solutions become more stable, the problem of equivalence is reduced, and the mean estimates of various parameters get closer to their true values. Therefore, the best results are obtained when all the four datasets are jointly inverted.

In the present analysis we have used the joint inversion algorithm under the assumption that for dissimilar datasets (e.g., DC and RFLS) the link factor between the two methods is geometry. However, joint inversion can be still more effective if a realistic relationship between resistivity and velocity is incorporated. While Dos Santos et al (1988) have tried to find empirical correlations between seismic velocity and electrical resistivity (Yale 1985), others have utilized fractal rock models (Bahr 1997; Spangenberg 1998; Kozlovskaya and Hjelt 2000) for this purpose. In yet another approach Greer et al (2002) have utilized an effective medium technique to integrate seismic and electromagnetic datasets. The analytical 

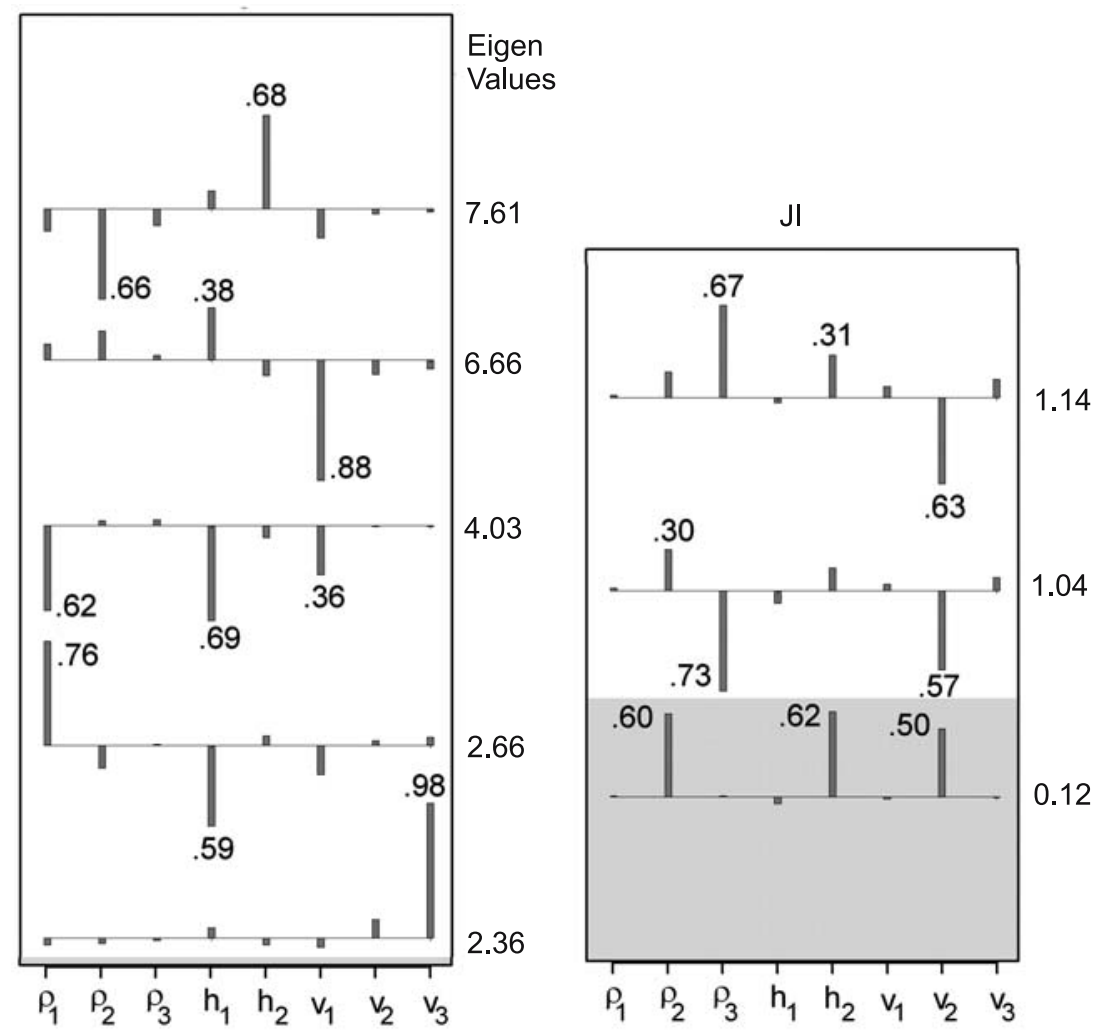

Figure 6. Model eigen-vector analysis for JI (joint inversion of all dataset) for the initial guess model IG-1.

relationship between the seismic macro-properties and electrical resistivity by such models could be utilized for effective optimization considering electrical and/or electromagnetic data jointly with seismic data.

\section{Acknowledgements}

We are grateful to the Director, National Geophysical Research Institute, for his encouragement and permission to publish the work. We would like to thank anonymous reviewers for their valuable comments and suggestions that helped in improving the manuscript.

\section{References}

Backus G and Gilbert F 1970 Uniqueness in the inversion of inaccurate gross earth data; Phil. Trans. Roy. Soc. London A 266 123-192.

Bahr K 1997 Electrical anisotropy and conductivity distribution functions of fractal random networks and of the crust: The scale effect of connectivity; Geophys. J. Int. $130649-660$.

Bailey R C 1970 Inversion of the geomagnetic induction problem; Proc. Roy. Soc. London A. 315 185-194.

Biswas S K 1982 Rift basins in the western margin of India and their hydrocarbon prospect; Bull. Am. Assoc. Pet. Geol. 66 1497-1513.
Dos Santos, Britto W L, Ulrych T J and deLima O A L 1988 A new approach to deriving pseudovelocity logs from resistivity logs; Geophys. Prosp. 36 83-91.

Gallardo L A and Meju M A 2007 Joint two-dimensional cross-gradient imaging of magnetotelluric and seismic travel time data for structural and lithological classification; Geophys. J. Int. 169 1261-1272.

Ghosh D P 1971 The application of linear filter theory to the direct interpretation of geoelectrical sounding measurements; Geophys. Prosp. 19 192-217.

Greer A A, Sinha M C and MacGregor L M 2002 Joint Effective Medium Modelling for Co-incident Seismic and Electromagnetic Data, and its Application to Studies of Porosity Structure at Mid-Ocean Ridge Crests; LITHOS Science Report 4 103-120.

Hering A, Misiek R, Gyulai A, Ormos T, Dobroka M and Dresen L 1995 A joint inversion algorithm to process geoelectric and surface wave seismic data, Part I: basic ideas; Geophys. Prosp. 43 135-156.

Inman J R 1973 Resistivity inversion; Geophysics 38 $1088-1108$.

Inman J R 1975 Resistivity inversion with ridge regression; Geophysics 40 798-817.

Jupp D L B and Vozoff K 1975 Stable iterative method for the inversion of geophysical data; Geophys. J. Roy. Astr. Soc. 42 952-976.

Koefoed O 1969 An analysis of equivalence in resistivity sounding; Geophys. Prosp. 17 327-335.

Kozlovskaya E and Hjelt S-E 2000 Modelling of elastic and electrical properties of solid liquid rock system with fractal microstructure; Phys. Chem. Earth, Part A: Solid Earth Geodesy 25 195-200.

Manglik A and Verma S K 1998 Delineation of sediments below flood basalts by joint-inversion of seismic 
and magnetotelluric data; Geophys. Res. Lett. 25 4015-4018.

Manglik A and Verma S K 1999 Delineation of fine sedimentary structures below Deccan Trap flood basalts employing a synergistic algorithm combining seismic, MT and DC resistivity data. In: Proc. of PETROTECH, New Delhi, Paper Nr. V4S3A10 (CD-Media).

Marquardt D W 1970 Generalised inverse, ridge regression, biased linear estimation and non-linear estimation; Technometrics 21 591-612.

Menke W 1989 Geophysical data analysis: Discrete inverse theory, Revised edition (San Diego: Academic Press) 289pp.

Milani E J, Kinoshita E M, Araujo L M and da Cruz Cunha P R 1990 Parana basins petroleum potential in the depocenter area; Boletim de Geociencias da Petrobra 4 21-34.

Morrison H F, Shoham Y, Hoversten G M and Torres-verdin C 1996 Electromagnetic mapping of electrical conductivity beneath the Columbia basalts; Geophys. Prosp. 44 963-986.

Sain K and Kaila K L 1994 Inversion of wide-angle seismic reflection times with damped least squares; Geophysics 59 1735-1744.

Sain K and Kaila K L 1996 Ambiguity in the solution to the velocity inversion problem and a solution by joint-inversion of seismic refraction and wide-angle reflection times; Geophys. J. Int. 124 215-217.
Satpal O P, Sar D, Chatterjee S M and Sawai S 2006 Integrated interpretation for sub-basalt imaging in Saurashtra Basin, India; The Leading Edge 25 882-885.

Sharma S P 1994 Resolution of fine 1-D structures employing individual and joint inversion of electrical and electromagnetic data. Ph.D. Thesis, Banaras Hindu University, $181 \mathrm{pp}$.

Spangenberg E 1998 A fractal model for physical properties of porous rock: Theoretical formulations and application to elastic properties; J. Geophys. Res. 103 $12,269-12,289$.

Verma S K and Sharma S P 1993 Resolution of thin layers using joint-inversion of electromagnetic and DC sounding data; J. EM Waves and Appl. 7 443-479.

Verma S K and Sri Niwas 1994 Comparison of the deep resistivity, magnetotelluric and deep TEM methods in the delineation of conducting sediments below high velocity lava flows; J. Assoc. Expl. Geophys. 15 31-39.

Vozoff K and Jupp D L B 1975 Joint inversion of geophysical data; Geophys. J. Roy. Astr. Soc. 42 977-991.

Warren R K 1996 A few case histories of subsurface imaging with EMAP as aid to seismic prospecting and interpretation; Geophys. Prosp. 44 923-934.

Withers R, Eggers D, Fox T and Crebs T 1994 A case study of integrated hydrocarbon exploration through basalt; Geophysics 59 1666-1679.

Yale D P 1985 Recent advances in rock physics; Geophysics $502480-2491$. 